ARTículo

\title{
Evaluación del proceso de gestión educativa para la integración de modelos didácticos mediados por TIC: un estudio de caso múltiple
}

\author{
Isabel Jiménez Becerra a \\ Kemel A. Ghotme ${ }^{b}$ \\ Artieres Estevao Romeiro ${ }^{c}$ \\ Lina Rosa Parra Bernal ${ }^{d}$
}

\section{Resumen}

El artículo presenta un estudio orientado a evaluar el efecto de la implementación de los modelos didácticos mediados por TIC, en las prácticas de Enseñanza en algunos contextos educativos colombianos, desde una investigación mixta enmarcada en los diseños de evaluación a partir de las teorías de Guskey para evaluar el efecto del fenómeno estudiado combinado con el diseño de investigación basado en el estudio caso múltiple que permite explicar el aporte de estos modelos en el aprendizaje. Se trabajó con 35 maestros colombianos de escuelas y universidades ubicadas en zonas geográficas rurales y urbanas, caracterizadas por pertenecer a escenarios con limitaciones económicas y realidades sociales marcadas por el conflicto. En las conclusiones fue posible corroborar la hipótesis alternativa (Ha) que declara que la gestión de proyectos de innovación educativa favorece parcialmente la cualificación de las prácticas de Enseñanza; esto, como producto de variables extrañas sobre la baja cantidad de dispositivos con las que cuenta la institución y los débiles conocimientos didácticos que posee el profesorado para el uso de las tecnologías en el aula.

Palabras clave: Modelos Didácticos. Gestión Educativa. Prácticas de Enseñanza. Evaluación.

\footnotetext{
a Universidad de La Sabana, Chía, Colombia.

b Universidad de La Sabana, Chía, Colombia.

c Universidad Técnica Particular de Loja, Loja, Ecuador.

d Universidad Católica de Manizales, Manizales, Colombia. 


\section{Introducción}

Trasladar la investigación aplicada al escenario de la gestión de proyectos educativos, con el objetivo de evaluar y avanzar en los procesos de innovación, es todo un reto. La raíz de esta labor es acudir a la naturaleza de la gestión y sus diversos tipos, germinando en sus participantes una cultura institucional, minada de logros comunes que favorezcan a la comunidad educativa en sus diversos campos de acción (académica, administrativa, directiva o comunitaria). Justamente desde ahí, la innovación se concibe como un acto de mejora, cuya dinámica se debate en innovar para transformar y transformar para mejorar.

Un componente alterno de la naturaleza de la gestión de proyectos, en opinión de Terribili Filho (2011), es que los proyectos han consolidado, desde su práctica, una ruta que permite ir más allá de la planificación; esto implica fundamentar, diseñar, implementar y evaluar la gestión de tales proyectos en los contextos educativos. Desde esta ruta, la evaluación (VOGLER; BLANCHARD; FIJALKOW, 1996) sobre la gestión de los proyectos educativos busca validar sus efectos ${ }^{1}$, los cuales, para el caso en particular de esta investigación, se relacionaron con el propósito de evaluar el proceso de gestión educativo para la integración de modelos didácticos mediados por la Tecnología de la Información y la Comunicación (en adelante TIC), implementados por los investigadores adscritos al proyecto de investigación "Didácticas Innovadoras mediadas por TIC: Retos a partir de la ecología del Aprendizaje" (JIMÉNEZ-BECERRA, SEGOVIA-CIFUENTES, 2020).

El proyecto ubicó conceptualmente a los investigadores ${ }^{2}$, al permitirles reconocer qué tipo de gestión educativa implementar, en el marco de los desafíos propuestos bajo el objetivo de identificar el uso de los elementos o procesos didácticos utilizados para lograr prácticas de Enseñanza exitosas (WESTRICK; MORRIS, 2016). Este producto emerge de la compilación hecha por Jiménez y Segovia (JIMÉNEZ-BECERRA, SEGOVIA-CIFUENTES, 2020), a partir del análisis sobre las finalidades y funciones de las técnicas de Enseñanza, las metodologías y su relación con la concepción de currículum de los investigadores adscritos al proyecto. De igual manera, a partir de la gestión institucional que implementaron

\footnotetext{
Considerando el efecto de la evaluación de todo comportamiento o acontecimiento del que puede, razonablemente, decirse que ha sido influido por algún aspecto del programa o proyecto (BOND, 1985 citado por JACOBY; MORA, 2008).

2 Cuando se habla de los investigadores adscritos se refiere a estudiantes posgraduales que realizaron programas de posgrados en la Universidad de La Sabana y que se vincularon, desde su interés en la investigación institucional, al proyecto señalado. También ubica a investigadores de Ecuador, Chile y otras universidades colombianas -algunos autores de este artículo- que apoyaron el análisis de los resultados de esta investigación y con quienes actualmente se investiga sobre "Modelos didácticos con mediación TIC: algunas implementaciones en las prácticas de enseñanza en profesores universitarios Latinoamericanos"
} 
para la formación y la aprehensión del profesorado en el uso de las TIC dentro de su quehacer pedagógico (KAPLAN; HAENLEIN, 2016; MILLER; SHAPIRO; HILDING-HAMANN, 2008).

Es de aclarar que este proceso le permitió a la investigadora principal identificar y proponer tres modelos de integración didáctica con TIC, en los que se articularon los investigadores en el marco de la fundamentación, diseño, implementación y evaluación de sus proyectos institucionales. Esta clasificación se presenta en tres niveles, a saber: primero, el uso de los recursos/técnicas TIC y las experiencias de aprendizaje; segundo, la concepción e implementación de las metodologías, y su coherencia con los enfoques curriculares; y tercero, un escenario didáctico centrado en la creación y uso de modelos que aportan a los componentes cognitivos necesarios para el aprendizaje.

En cada tipología se analizaron componentes relacionados con los criterios de inclusión que concibe el profesorado para su uso; también su relación con el escenario de Enseñanza que posibilita experiencias de aprendizaje específicas. Los modelos didácticos que pueden ser vistos como los desafíos de la Educación con TIC son expuestos en la Figura 1.

Figura 1 - Desafíos del proyecto Didácticas Innovadoras mediadas por TIC

DIDÁCTICAS INNOVADORAS MEDIADAS POR TIC: RETOS A PARTIR DE LA ECOLOGÍA DEL APRENDIZAJE

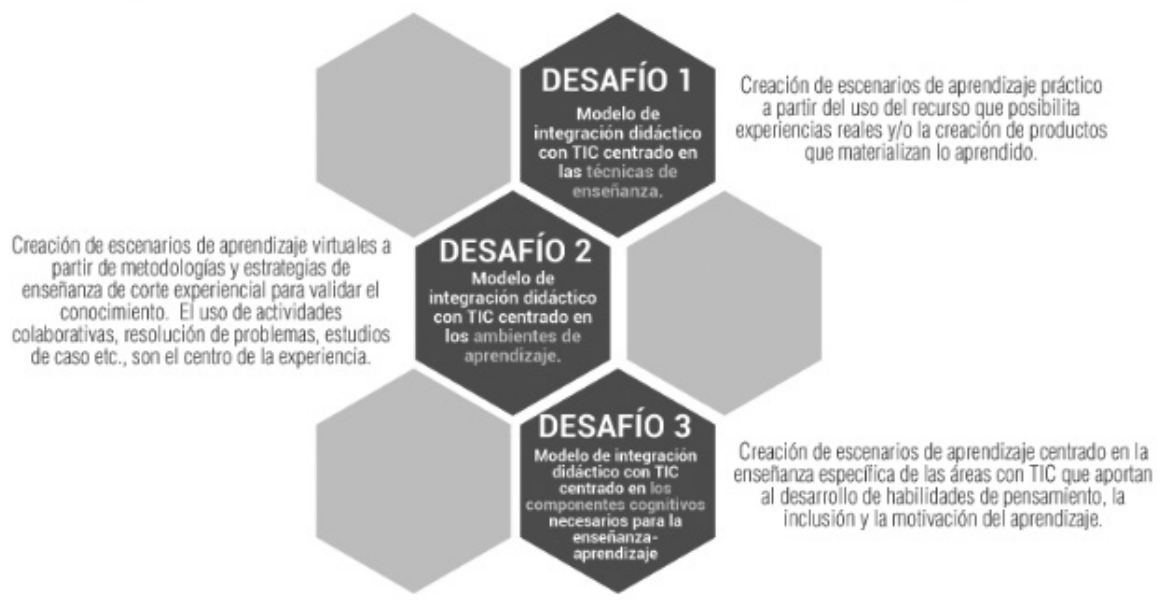

Fuente: Jiménez-Becerra; Segovia-Cifuentes (2020) 
Entonces, se hace necesario ajustar y examinar, de manera crítica, diferentes asuntos concernientes a la formación, a la profesionalización y a la ejecución docente, así como a las estrategias para generar procesos de gestión y transformación que aporten a esta ecuación de calidad educativa (BRASLAVSKY, 1999; CARDELLI; DUHALDE, 2001; SUMMO; VOISIN; TÉLLEZ-MÉNDEZ, 2016). En efecto, esta es la base del proyecto y lo que sustenta el interés de los investigadores, quienes buscaron responder a la pregunta ¿cómo evaluar el efecto del proceso de gestión educativa para la integración de modelos didácticos mediados por TIC en los contextos educativos colombianos?

\section{Metodología}

La problematización del proyecto Didácticas Innovadoras mediadas por TIC centrado en ¿cómo replantear el papel de la didáctica a partir de los retos que plantea la ecología del aprendizaje mediado por las TIC? generó objetivos como identificar las concepciones didácticas de los maestros respecto al uso las TIC (JIMÉNEZ-BECERRA, SEGOVIA-CIFUENTES, 2020). Además, reconocer y aplicar algunos modelos de integración didáctica con mediación TIC, que aporten al replanteamiento de la didáctica en el marco de la ecología del aprendizaje (COLL SALVADOR, 2013; JIMÉNEZ-BECERRA, 2020a).

En cuanto a los investigadores participantes, fueron 35 maestros colombianos de 16 instituciones educativas ubicados en zonas geográficas urbanas y rurales de Colombia, caracterizadas por pertenecer a contextos con limitaciones económicas y realidades sociales marcadas por el conflicto. Es de anotar que los casos presentados en esta investigación se acopiaron así: 35 investigadores, de los cuales 32 estaban en la región de Cundinamarca y, por ende, pertenecían a la misma Secretaría Gubernamental de Educación, implementando sus prácticas de Básica Primaria y Secundaria. De manera alterna se articularon, para esta investigación, 3 investigadores más quienes estaban ubicados en zonas apartadas de Cundinamarca, como Amazonas, Quindío, Valle del Cauca. La unión de sus equipos docentes para su implementación compiló un total de 150 profesores ubicados en estas 16 instituciones. Su participación, por estar en otras regiones del país, no fue un impedimento, recordando que esta investigación se centró en indagar por la formación del profesorado en tales modelos usando las TIC sin importar el tipo de población a la que imparte sus procesos de Enseñanza: primaria, secundaria o Educación Superior o su lugar geográfico de ubicación.

Dentro de la investigación se utilizó una metodología mixta, tanto por el uso combinado de diseños, como por instrumentos aplicados para la implementación, 
y por el modelo de evaluación de Guskey (1986) y el estudio caso único (STAKE, 1999), desde donde se logró evaluar el proceso de gestión educativo para la integración de modelos didácticos mediados por TIC. El estudio de caso múltiple descifró cómo se adoptó la integración de modelos didácticos mediados por TIC por parte de los docentes que integraron las 15 unidades de análisis ${ }^{3}$. En los diagnósticos institucionales estos participantes coincidieron en que su problemática está relacionada con esa realidad, siendo este el criterio para clasificarlas en una serie de unidades o subunidades cuya caracterización exigió de un tratamiento diferenciado (JIMÉNEZ-BECERRA, 2020a).

Por su parte, el modelo de Guskey (1986) permitió evaluar los procesos de gestión educativa implementados por los investigadores integrantes del proyecto, desde la propia identificación de que el cambio en los conocimientos, las actitudes y las creencias del profesor no se llevaría a cabo sino hasta que cada individuo comprendiera que la transformación de la práctica de aula condujo a un provecho estudiantil adecuado. Para ello se utilizaron técnicas de investigación como las encuestas, las entrevistas y los grupos focales. Respecto a su validación, se utilizaron tanto instrumentos ya validados e implementados en otras investigaciones y que fueron facilitadas por los investigadores, previo consentimiento, como instrumentos nuevos, que fueron validados por juicio de expertos que revisaron y apoyaron, con su evaluación, la cualificación de los mismos.

A partir de esta propuesta, el tratamiento diferenciado de la información para ser evaluada se planteó en dos grupos de análisis mediante cuatro tipos de indicadores. Los dos primeros indicadores, enmarcados en el diseño de estudio de caso (STAKE, 1999) y clasificados como proceso, se centraron en analizar y descifrar el interés de los investigadores por el tipo de modelo didáctico que orientaría la construcción del prototipo para operacionalizar como piloto, mostrando en ellos el valor de su gestión en las instituciones focalizadas.

El segundo grupo de indicadores enmarcados en el resultado y el producto fueron ubicados dentro del diseño de evaluación aplicada al modelo Guskey (1986). De este modo, se recopilaron resultados sobre el número de profesores capacitados en la institución y cuántos de ellos implementaron el uso de los modelos didácticos en sus prácticas de Enseñanza con mediación TIC. Los componentes metodológicos de la investigación se resumen en la Tabla 1.

3 Se refiere a las 15 instituciones en las cuales se implementó la investigación. 
Tabla 1 - Diseño metodológico de las investigaciones articuladas al proyecto ${ }^{4}$

\begin{tabular}{|c|c|c|c|c|}
\hline Proyecto & \multicolumn{4}{|c|}{$\begin{array}{c}\text { Didácticas Innovadoras mediadas por TIC: retos a partir de la ecología } \\
\text { del aprendizaje }\end{array}$} \\
\hline $\begin{array}{l}\text { Gestión de } \\
\text { Proyectos }\end{array}$ & \multicolumn{4}{|c|}{$\begin{array}{c}\text { Evaluación del proceso de gestión educativa para la integración de modelos } \\
\text { didácticos mediados por TIC }\end{array}$} \\
\hline Pregunta & \multicolumn{4}{|c|}{$\begin{array}{c}\text { ¿Cómo evaluar el efecto del proceso de gestión educativo para la } \\
\text { integración de modelos didácticos mediados por TIC en los contextos } \\
\text { educativos colombianos? }\end{array}$} \\
\hline \multirow{2}{*}{$\begin{array}{l}\text { Objetivos de } \\
\text { efecto }\end{array}$} & Proceso & \multicolumn{3}{|c|}{$\begin{array}{c}\text { Analizar y descifrar las concepciones de uso de los } \\
\text { modelos didácticos con mediación TIC en las prácticas de } \\
\text { Enseñanza a partir de los criterios de inclusión requeridos } \\
\text { para el aprendizaje }\end{array}$} \\
\hline & $\begin{array}{l}\text { Resultado y } \\
\text { producto }\end{array}$ & \multicolumn{3}{|c|}{$\begin{array}{l}\text { Evaluar el efecto de la implementación de los modelos } \\
\text { didácticos mediados por TIC en las prácticas de } \\
\text { Enseñanza de los profesores objeto de estudio }\end{array}$} \\
\hline \multirow{7}{*}{ Diseño Mixto } & \multirow{2}{*}{$\begin{array}{l}\text { Mixto por } \\
\text { diseños }\end{array}$} & \multicolumn{3}{|c|}{ Evaluación aplicada Modelo de Evaluación Guskey (1986) } \\
\hline & & \multicolumn{3}{|c|}{ Estudio de caso múltiple (Stake, 1999) } \\
\hline & \multirow{5}{*}{$\begin{array}{l}\text { Mixto por } \\
\text { técnicas e } \\
\text { instrumentos }\end{array}$} & Técnica & Instrumento & Tipo de datos \\
\hline & & Cuestionario & Encuesta & Cuantitativos \\
\hline & & Entrevista & Cuestionario & Cualitativos \\
\hline & & Cuestionario & $\begin{array}{l}\text { Guía de } \\
\text { entrevista del } \\
\text { Grupo Focal }\end{array}$ & Cualitativos \\
\hline & & Observación & Lista de chequeo & Cualitativo \\
\hline Alcance & \multicolumn{4}{|c|}{ Explicativo } \\
\hline Población & \multicolumn{4}{|c|}{$\begin{array}{l}\text { Profesores con prácticas de Enseñanza en Educación Básica, Media y } \\
\text { Superior colombianos }\end{array}$} \\
\hline Muestra & \multicolumn{4}{|c|}{ Participación de 150 profesores agrupados en las 15 instituciones } \\
\hline \multirow{3}{*}{ Contextos } & Quindío & Valle del Cauca & Cundinamarca & Amazonas \\
\hline & \#1 & \#1 & $\# 13$ & $\# 1$ \\
\hline & $\begin{array}{l}\text { Educación } \\
\text { Superior }\end{array}$ & $\begin{array}{l}\text { Educación } \\
\text { Secundaria }\end{array}$ & $\begin{array}{l}\text { Educación } \\
\text { Básica Primaria y } \\
\text { Secundaria }\end{array}$ & Básica Secundaria \\
\hline \multirow[b]{2}{*}{ Variables } & \multicolumn{2}{|c|}{ Dependiente } & \multicolumn{2}{|c|}{$\begin{array}{l}\text { Transformación de prácticas de } \\
\text { Enseñanza }\end{array}$} \\
\hline & \multicolumn{2}{|c|}{ Independiente } & \multicolumn{2}{|c|}{$\begin{array}{l}\text { Implementación de modelos de } \\
\text { integración didácticos mediados por } \\
\text { TIC para la innovación }\end{array}$} \\
\hline
\end{tabular}

Continua

\footnotetext{
4 Cuadro metodológico "ensamblado", concepto usado por Jiménez (2020a) para presentar en una misma estructura todos los componentes de la investigación.
} 
Continuación

\begin{tabular}{|c|c|c|c|c|c|}
\hline \multirow{3}{*}{$\begin{array}{l}\text { Control de } \\
\text { Variables }\end{array}$} & \multicolumn{5}{|c|}{$\begin{array}{c}\text { La gestión de proyectos de innovación educativa para la implementación } \\
\text { de modelos de integración didácticos mediados por TIC favorece la } \\
\text { cualificación de las prácticas de Enseñanza }\end{array}$} \\
\hline & \multicolumn{5}{|c|}{$\begin{array}{c}\text { La gestión de proyectos de innovación educativa para la } \\
\text { implementación de modelos de integración didácticos mediados } \\
\text { por TIC favorece parcialmente la cualificación de las prácticas } \\
\text { de Enseñanza }\end{array}$} \\
\hline & \multicolumn{5}{|c|}{$\begin{array}{c}\text { La gestión de proyectos de innovación educativa para la implementación } \\
\text { de modelos de integración didácticos mediados por TIC es indiferente en } \\
\text { la transformación de las prácticas de Enseñanza }\end{array}$} \\
\hline \multirow{3}{*}{ Hipótesis } & Hi & \multicolumn{4}{|c|}{$\begin{array}{c}\text { La gestión de proyectos de innovación educativa } \\
\text { para la implementación de modelos de integración didácticos } \\
\text { mediados por TIC favorece la cualificación de las prácticas } \\
\text { de Enseñanza }\end{array}$} \\
\hline & $\mathrm{Ha}$ & \multicolumn{4}{|c|}{$\begin{array}{l}\text { La gestión de proyectos de innovación educativa para la } \\
\text { implementación de modelos de integración didácticos mediados } \\
\text { por TIC favorece parcialmente la cualificación de las prácticas } \\
\text { de Enseñanza }\end{array}$} \\
\hline & Ho & \multicolumn{4}{|c|}{$\begin{array}{l}\text { La gestión de proyectos de innovación educativa para la } \\
\text { implementación de modelos de integración didácticos mediados } \\
\text { por TIC es indiferente en la transformación de las prácticas } \\
\text { de Enseñanza }\end{array}$} \\
\hline & \multicolumn{3}{|c|}{$\begin{array}{l}\text { Estudio de caso múltiple } \\
\text { (STAKE, 1999). }\end{array}$} & \multicolumn{2}{|c|}{ Modelo de Evaluación Guskey (1986). } \\
\hline $\begin{array}{l}\text { Objetivos de } \\
\text { efectos }\end{array}$ & \multicolumn{3}{|c|}{$\begin{array}{c}\text { Analizar y descifrar las } \\
\text { concepciones de uso de los } \\
\text { modelos didácticos con } \\
\text { mediación TIC en las prácticas } \\
\text { de Enseñanza a partir de los } \\
\text { criterios de inclusión requeridos } \\
\text { para el aprendizaje }\end{array}$} & \multicolumn{2}{|c|}{$\begin{array}{c}\text { Evaluar el efecto de la } \\
\text { implementación de los modelos } \\
\text { didácticos mediados por TIC en } \\
\text { las prácticas de Enseñanza de los } \\
\text { profesores objeto de estudio }\end{array}$} \\
\hline $\begin{array}{l}\text { Fases del } \\
\text { Proyecto } \\
\text { Educativo }\end{array}$ & \multicolumn{2}{|c|}{ Fundamentación } & Diseño & \multicolumn{2}{|c|}{ Implementación y evaluación } \\
\hline Objetivos & $\begin{array}{l}\text { Diag } \\
\text { estad } \\
\text { de } E\end{array}$ & $\begin{array}{l}\text { nosticar el } \\
\text { o prácticas } \\
\text { nseñanza }\end{array}$ & $\begin{array}{l}\text { Prototipar } \\
\text { el modelo } \\
\text { didáctico con } \\
\text { mediación TIC a } \\
\text { implementar }\end{array}$ & $\begin{array}{l}\text { Evaluar el aporte } \\
\text { del prototipo en la } \\
\text { gestión educativa } \\
\text { y académica. }\end{array}$ & $\begin{array}{l}\text { Reconocer los } \\
\text { resultados } \\
\text { y pertinencia } \\
\text { para la } \\
\text { implementación } \\
\text { en los contextos }\end{array}$ \\
\hline
\end{tabular}

Continua 
Continuación

\begin{tabular}{|c|c|c|c|c|}
\hline \multirow{3}{*}{$\begin{array}{l}\text { Tipos de } \\
\text { Indicadores }\end{array}$} & \multicolumn{2}{|c|}{ Proceso } & \multirow[b]{2}{*}{$\begin{array}{c}\text { Resultado } \\
90 \text { \# de } \\
\text { profesores } \\
\text { capacitados } \\
\text { para implementar } \\
\text { tipos de modelos } \\
\text { de integración } \\
\text { didácticos } \\
\text { mediados por } \\
\text { TIC entre el } \\
\text { año } 2015 \\
\text { al } 2018\end{array}$} & \multirow[b]{2}{*}{$\begin{array}{c}\text { Productos } \\
15 \text { \# de modelos } \\
\text { de integración } \\
\text { didácticos } \\
\text { mediados } \\
\text { por TIC } \\
\text { articulados a } \\
\text { las prácticas de } \\
\text { Enseñanza } \\
\text { de profesores } \\
\text { entre el } \\
\text { año } 2015 \text { al } 2018\end{array}$} \\
\hline & $\begin{array}{c}150 \# \text { de } \\
\text { profesores } \\
\text { interesados } \\
\text { en la } \\
\text { cualificación } \\
\text { las prácticas de } \\
\text { Enseñanza con } \\
\text { mediación TIC } \\
\text { entre el año } \\
2015 \text { al } 2018\end{array}$ & $\begin{array}{l}15 \text { \# prototipos } \\
\text { de modelos } \\
\text { de integración } \\
\text { didácticos } \\
\text { mediados por } \\
\text { TIC propuestos } \\
\text { por los } \\
\text { investigadores } \\
\text { entre el año } 2015 \\
\text { al } 2018\end{array}$ & & \\
\hline & $\begin{array}{l}150 \# \text { de } \\
\text { profesores } \\
\text { inscritos }\end{array}$ & $\begin{array}{l}15 \text { \# prototipos } \\
\text { propuestos }\end{array}$ & $\begin{array}{c}150 \# \text { de } \\
\text { profesores } \\
\text { interesados } \\
\text { capacitarse }\end{array}$ & $\begin{array}{l}15 \text { \# de modelos } \\
\text { implementados }\end{array}$ \\
\hline $\begin{array}{l}\text { Instrumentos } \\
\text { de recolección } \\
\text { de datos por } \\
\text { fases }\end{array}$ & \multicolumn{2}{|c|}{ Encuesta } & \multicolumn{2}{|c|}{$\begin{array}{c}\text { Grupos Focales -Entrevista - } \\
\text { Cuestionario }\end{array}$} \\
\hline
\end{tabular}

Fuente: Elaboración propia (2020)

La información obtenida permitió monitorear tanto los procesos como los resultados que, en el marco de la Educación, buscan apuntar a seguir cualificando la calidad educativa a partir del uso de las TIC, cuyos orígenes son la reflexión y transformación de las prácticas de Enseñanza. Es de anotar que la evaluación permanente (REMOLINA-CAVIEDES, 2016) en la implementación de estos proyectos institucionales permitió reconocer cómo transformar las prácticas de Enseñanza usando TIC, en concordancia con las necesidades del contexto social y la demanda sobre los tipos de aprendizaje que requieren los estudiantes para cualificar su proceso, aspecto socavado de los datos extraídos.

\section{Resultados}

En este apartado se exponen los resultados de investigación analizados a partir de los datos socavados por instrumentos en cada fase del proyecto educativo, en sinergia con el objetivo a evaluar y los indicadores a medir. 


\subsection{Fases fundamentación y diseño del proyecto educativo}

El objetivo de esta fase fue analizar y descifrar las concepciones de uso de los modelos didácticos con mediación TIC en las prácticas de Enseñanza a partir de los criterios de inclusión requeridos para el aprendizaje. Para lograr el mismo, se aplicaron dos experiencias prácticas: la primera de ellas, generar un diagnóstico institucional sobre las prácticas de Enseñanza con mediación TIC, identificando la concepción y alcance que posee el profesorado objeto de estudio en sus prácticas de Enseñanza, así como las características que tenía cada institución para su implementación. La recolección de datos de esta fase se generó a partir del uso de instrumentos como el cuestionario de acceso al campo aplicado a los profesores de las instituciones.

Los núcleos más destacados en este cuestionario, validado desde la matriz TIC (LUGO; KELLY, 2011), giraron en cuatro aspectos: las iniciativas que ha tenido la institución respecto al uso de las TIC para la Enseñanza, los tipos de escenarios TIC usados, y la concepción de su uso en la planeación e implementación didáctica de sus clases la existencia de los recursos humanos y de infraestructura para su implementación. Los resultados que se presentan compilan los resultados del indicador declarado como proceso y poseen la siguiente parametrización (Tabla 2).

Tabla 2 - Indicador proceso

150 \# de profesores interesados en la cualificación las prácticas de Enseñanza con mediación TIC entre el año 2015 al 2018

150 \# de profesores inscritos

Fuente: Elaboración propia (2020)

La estrategia para analizar los resultados del cuestionario realizado a los 150 participantes se declaró en la matriz Dofa: debilidades, oportunidades, fortalezas y amenazas generando un diagnóstico de las instituciones para, posteriormente, intervenir en la formulación e implantación de estrategias así como su seguimiento para efectos de evaluación y control (REMOLINA, 2016). A partir de esta intención, se graficaron los resultados del diagnóstico en el árbol de problemas expuesto en la Figura 2 (MARTÍNEZ; FERNÁNDEZ, 2008). 
Figura 2 - Definición del Problema Educativo: Resultados del Dofa

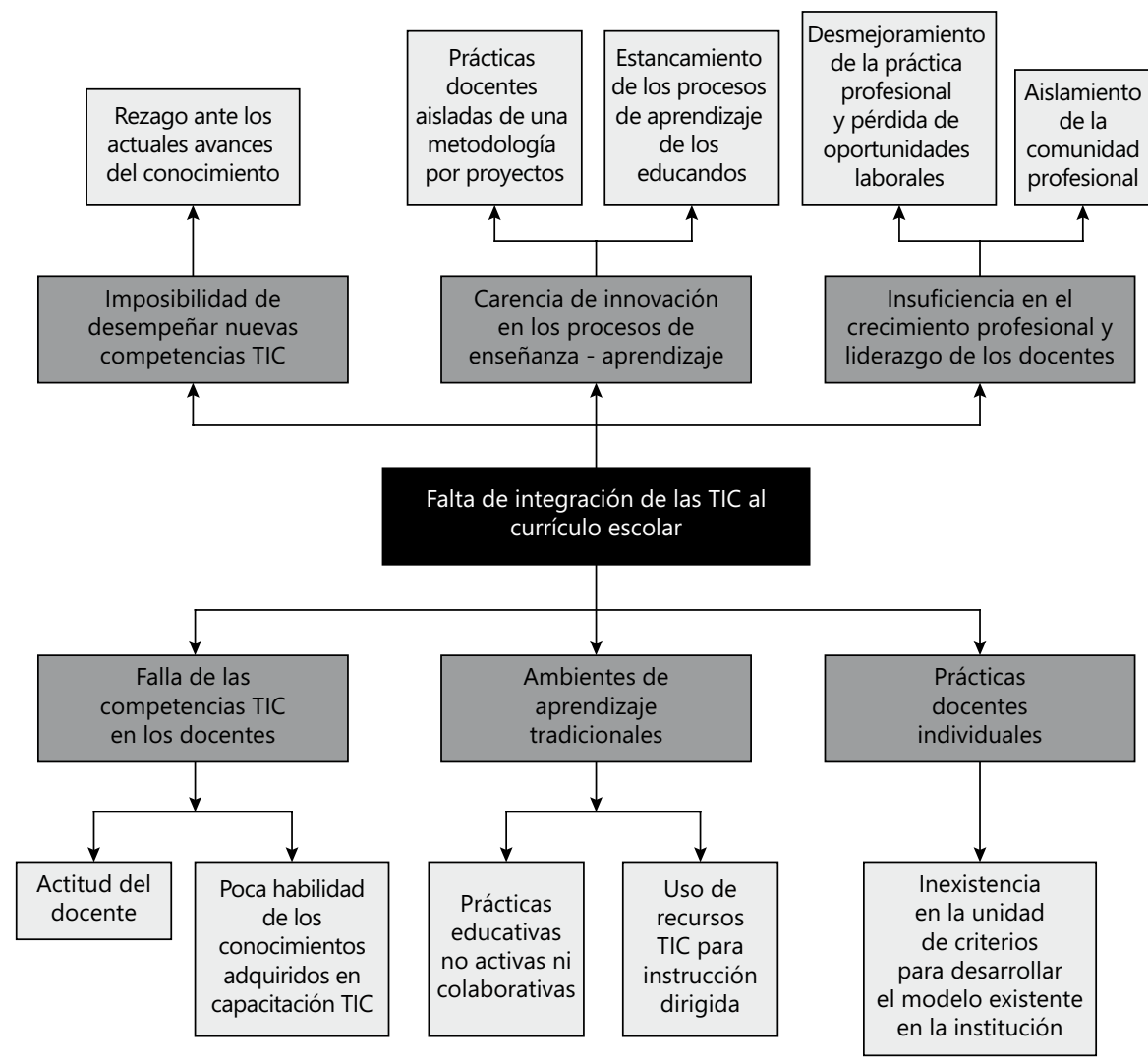

Fuente: Londoño, Rubiano y Triana (2018, p. 33)

Según Londoño, Rubiano y Triana (2018), investigadores encargados de consolidar este diagnóstico, los resultados observados permitieron analizar y descifrar las concepciones de uso de los modelos didácticos con mediación TIC, en las prácticas de Enseñanza a partir de los criterios de inclusión requeridos para el aprendizaje. Esto fue posible al identificar que los profesores reconocen la necesidad de una integración de las TIC a las prácticas de Enseñanza, a través del currículo. Otro aspecto para considerar es la inexistencia de competencias TIC en los docentes para reconocer las estrategias didácticas con mediación tecnológica.

El cuanto a los criterios de inclusión, el profesorado plantea que los ambientes de aprendizaje continúan siendo tradicionales (HERRERA; FERNÁNDEZ; SEGUEL, 
2018) y, por ende, las prácticas carecen de actividades que despierten el interés hacia el conocimiento, dado que no hay claridad en la selección de material o una metodología específica. Asimismo, el uso de recursos TIC no se encuentra inmerso dentro de sus estrategias metodológicas por falta de capitación a nivel de saberes didácticos y competencias digitales.

Por esta razón, las prácticas de Enseñanza no responden a un direccionamiento que apunte a la unidad de criterios, acordes con el modelo de aprendizaje significativo definido en la misión y visión de sus instituciones, siendo esta un ejemplo de alcance del aprendizaje en sus estudiantes. Se constata poco uso de los recursos tecnológicos produciendo un rezago respecto a las competencias tecnológicas (DONG; NEWMAN, 2016), tales como manejo, producción e intercambio de información, creación de contenido y alfabetización TIC.

Como segundo momento de esta fase, se centró en proponer los prototipos de modelo didáctico con mediación TIC más pertinente a implementar en sus instituciones a partir de los resultados mapeados como la carencia de innovación en los procesos de Enseñanza y aprendizaje y los tipos modelos didácticos. Como resultado se obtuvieron 15 prototipos (uno a implementar por institución), siendo medibles y comprobables en el siguiente indicador (Tabla 3).

Tabla 3 - Indicador proceso

15 \# prototipos de modelos de integración didácticos mediados por TIC propuestos por los investigadores entre el año 2015 al 2018

15 \# prototipos propuestos

Fuente: Elaboración propia (2020)

Fue afianzada su elección por las teorías generadas dentro del proyecto Didácticas Innovadoras mediadas por TIC: Retos a partir de la ecología del aprendizaje (JIMÉNEZ-BECERRA; SEGOVIA-CIFUENTES, 2020), donde ya se contaban con tres tipologías o modelos a adoptar. A continuación, se presenta un cuadro comparativo que compila algunos prototipos creados por los investigadores ${ }^{5}$ participantes, tipificaciones por Jiménez-Becerra y Segovia-Cifuetes (2020), que permitió construir los mismos basado en el criterio de uso (Tabla 4).

Todas las investigaciones que se articulan a estos resultados se ubican en la base de datos "Intellectum" de la Universidad de La Sabana: https://intellectum.unisabana.edu.co/handle/10818/9 
Tabla 4 - Prototipos de Modelos didácticos con mediación TIC a implementar en los contextos educativos

\begin{tabular}{|c|c|c|c|c|}
\hline Tipo & Desafío & $\begin{array}{c}\text { Criterios de } \\
\text { inclusión }\end{array}$ & $\begin{array}{c}\text { Algunos ejemplos de } \\
\text { prototipo }\end{array}$ & Contexto \\
\hline \multirow{4}{*}{$\begin{array}{l}\text { Modelo de } \\
\text { integración } \\
\text { didáctico } \\
\text { con TIC } \\
\text { centrado } \\
\text { en las } \\
\text { técnicas de } \\
\text { Enseñanza }\end{array}$} & \multirow{4}{*}{$\begin{array}{l}\text { Creación de } \\
\text { recursos que } \\
\text { favorezcan el } \\
\text { aprendizaje } \\
\text { para } \\
\text { experimentar } \\
\text { situaciones } \\
\text { reales: } \\
\text { autogestión del } \\
\text { aprendizaje y } \\
\text { generación de } \\
\text { experiencias } \\
\text { de simulación } \\
\text { centradas en } \\
\text { problemáticas } \\
\text { sociales }\end{array}$} & $\begin{array}{l}\text { Entornos } \\
\text { Virtuales de } \\
\text { Aprendizaje } \\
\text { o Personal } \\
\text { Learning } \\
\text { Environments } \\
\text { (PLE) }\end{array}$ & $\begin{array}{c}\text { Implementación } \\
\text { de entornos } \\
\text { personales de } \\
\text { aprendizaje para } \\
\text { fortalecer las habilidades } \\
\text { comunicativas. } \\
\text { Folios, 47, 119-132. } \\
\text { (JIMÉNEZ-BECERRA; } \\
\text { SALAMANCA } \\
\text { ESPOINOSA; LÓPEZ; } \\
\text { LÓPEZ 2018) }\end{array}$ & Cundinamarca \\
\hline & & \multirow{3}{*}{$\begin{array}{c}\text { Materiales } \\
\text { Educativos } \\
\text { Digitales }\end{array}$} & $\begin{array}{l}\text { Recurso educativo } \\
\text { digital adaptativo "el } \\
\text { rescate del reino": una } \\
\text { estrategia para aprender } \\
\text { permutaciones sin } \\
\text { repetición } \\
\text { (VILLARREAL, 2016) }\end{array}$ & Cundinamarca \\
\hline & & & $\begin{array}{c}\text { Creación de } \\
\text { Materiales para Recursos } \\
\text { Educativos Digitales } \\
\text { Abiertos (REDA): } \\
\text { una Estrategia de } \\
\text { Aprendizaje por } \\
\text { Proyectos que } \\
\text { aporta al Desarrollo } \\
\text { de Pensamiento } \\
\text { Computacional en } \\
\text { el ciclo de Educación } \\
\text { Media en la } \\
\text { Escuela Normal } \\
\text { Superior de Leticia } \\
\text { (ERICSON, 2018) }\end{array}$ & Amazonas \\
\hline & & & $\begin{array}{l}\text { Uso didáctico del } \\
\text { video juego para } \\
\text { la Enseñanza de las } \\
\text { Ciencias Sociales: } \\
\text { un estado en cuestión } \\
\text { (JIMÉNEZ-BECERRA; } \\
\text { ESCOBAR, 2016) }\end{array}$ & Cundinamarca \\
\hline
\end{tabular}

Continua 


\begin{tabular}{|c|c|c|c|c|}
\hline \multirow{3}{*}{$\begin{array}{l}\text { Modelo de } \\
\text { integración } \\
\text { didáctico } \\
\text { con TIC a } \\
\text { partir de los } \\
\text { ambientes } \\
\text { de } \\
\text { aprendizaje }\end{array}$} & \multirow{3}{*}{$\begin{array}{l}\text { Creación de } \\
\text { escenarios de } \\
\text { aprendizaje } \\
\text { virtuales } \\
\text { donde las } \\
\text { metodologías } \\
\text { presentan una } \\
\text { conexión con } \\
\text { los recursos TIC } \\
\text { para desarrollar } \\
\text { actividades } \\
\text { colaborativas, } \\
\text { resolución de } \\
\text { problemas, } \\
\text { estudios de } \\
\text { caso etc. }\end{array}$} & Metodologías & $\begin{array}{c}\text { Estrategias didácticas } \\
\text { mediadas por lego }{ }^{\circledR} \\
\text { mindstorms }{ }^{\circledR} \text { para } \\
\text { desarrollar el pensamiento } \\
\text { lógico para la programación } \\
\text { en los estudiantes de la } \\
\text { facultad de ingeniería de } \\
\text { la Universidad del Quindío } \\
\text { (ALDANA; BUITRAGO, 2019) }\end{array}$ & Quindío \\
\hline & & $\begin{array}{l}\text { Estrategias } \\
\text { didácticas }\end{array}$ & $\begin{array}{l}\text { Influencia de un entorno } \\
\text { personal de aprendizaje } \\
\text { en procesos de búsqueda, } \\
\text { selección y evaluación } \\
\text { de información para los } \\
\text { proyectos de investigación } \\
\text { de los estudiantes del } \\
\text { curso } 1002 \text { del colegio } \\
\text { Marco Antonio Carreño } \\
\text { Silva IED (CASALLAS, 2016) }\end{array}$ & \multirow[b]{2}{*}{ Cundinamarca } \\
\hline & & $\begin{array}{c}\text { Diseños de } \\
\text { secuencias } \\
\text { esquematizadas } \\
\text { por temáticas o } \\
\text { módulos }\end{array}$ & $\begin{array}{c}\text { Implementación } \\
\text { de un programa de } \\
\text { aprovechamiento positivo } \\
\text { del tiempo libre mediado } \\
\text { por TIC en los estudiantes } \\
\text { de la básica secundaria } \\
\text { y media de la Institución } \\
\text { Educativa Fonquetá } \\
\text { del municipio de Chía, } \\
\text { Cundinamarca (CHITIVA; } \\
\text { TORRES, 2018) }\end{array}$ & \\
\hline \multirow{2}{*}{$\begin{array}{l}\text { Modelo de } \\
\text { integración } \\
\text { con TIC } \\
\text { centrado } \\
\text { en los } \\
\text { componentes } \\
\text { cognitivos } \\
\text { necesarios } \\
\text { para la } \\
\text { Enseñanza - } \\
\text { aprendizaje }\end{array}$} & \multirow{2}{*}{$\begin{array}{l}\text { Creación de } \\
\text { escenarios de } \\
\text { aprendizaje } \\
\text { centrados en } \\
\text { la Enseñanza } \\
\text { específica } \\
\text { de las áreas, } \\
\text { para lograr el } \\
\text { desarrollo de } \\
\text { habilidades de } \\
\text { pensamiento, } \\
\text { la inclusión y la } \\
\text { motivación del } \\
\text { aprendizaje }\end{array}$} & $\begin{array}{l}\text { Desarrollo del } \\
\text { pensamiento } \\
\text { científico y sus } \\
\text { habilidades por } \\
\text { disciplinas }\end{array}$ & $\begin{array}{c}\text { Aprendizaje para la } \\
\text { Comprensión: estrategias } \\
\text { didácticas para fomentar } \\
\text { el pensamiento } \\
\text { matemático a partir de un } \\
\text { Ambiente de aprendizaje } \\
\text { Colaborativo mediado } \\
\text { por TIC en los IED San } \\
\text { Francisco y Manuelita } \\
\text { Sáenz (SÁNCHEZ; GALVIS, } \\
\text { 2017) }\end{array}$ & \multirow[t]{2}{*}{ Cundinamarca } \\
\hline & & $\begin{array}{l}\text { Inclusión } \\
\text { educativa }\end{array}$ & $\begin{array}{l}\text { Propuesta pedagógica } \\
\text { mediada por TIC para } \\
\text { fortalecer el proceso } \\
\text { de lectura y escritura } \\
\text { inicial en estudiantes } \\
\text { con diagnóstico con } \\
\text { necesidades educativas } \\
\text { especiales (GÓMEZ, 2018) }\end{array}$ & \\
\hline
\end{tabular}

Fuente: Elaboración propia (2020) 
Como se evidencia en la Tabla 4, los modelos seleccionados (JIMÉNEZBECERRA; SEGOVIA-CIFUENTES, 2020), los criterios que se tuvieron en cuenta por los investigadores para su elección se basó en las transformaciones de las situaciones didácticas problémicas detectadas así como los criterios de inclusión que concibe el profesorado para su uso así como su relación con las experiencias de aprendizaje posibles y el efecto en este proceso (JIMÉNEZ-BECERRA; FERNÁNDEZ PALMA; ALMENÁREZ MORENO, 2020).

\subsection{Fases implementación y evaluación proyecto educativo}

El objetivo de esta fase fue evaluar el efecto de la implementación de los modelos didácticos mediados por TIC evaluando el aporte del prototipo en las prácticas de Enseñanza en las instituciones seleccionadas. Para la recolección de datos, se utilizó un cuestionario aplicado posterior a la implementación, así como un conjunto de preguntas de percepción al profesorado que permitió descifrar su experiencia al aplicar el Ambiente Virtual (AAV) o el Recurso Educativo Digital De Aprendizaje (Reda).

Los indicadores para evaluar esta fase fueron resultado y producto, logrando medir el efecto de la integración y uso de los 15 prototipos en las prácticas de Enseñanza. Se contó con la participación solo de 90 profesores de los 150 que iniciaron la experiencia, dado que no todos los profesores que participaron en la primera fase de esta investigación, como muestra poblacional, contaban con los tiempos requeridos para el pilotaje (Tabla 5).

Tabla 5 - Indicador proceso

\begin{tabular}{|c|c|}
\hline $\begin{array}{l}90 \text { \# de profesores capacitados para } \\
\text { implementar tipos de modelos de } \\
\text { integración didácticos mediados por TIC } \\
\text { entre el año } 2015 \text { al } 2018\end{array}$ & $\begin{array}{c}15 \text { \# de modelos de integración didácticos } \\
\text { mediados por TIC articulados a las prácticas } \\
\text { de Enseñanza de profesores entre el año } \\
2015 \text { al } 2018\end{array}$ \\
\hline 150 \# de profesores interesados capacitarse & 15 \# de modelos implementados \\
\hline
\end{tabular}

Fuente: Elaboración propia (2020)

Frente a los resultados obtenidos, en la Tabla 6 donde se presenta el número de prototipos implementados por modelo didáctico de mediación TIC en sinergia con la percepción del profesorado producto de los datos obtenidos en el cuestionario, se evaluaron los criterios de inclusión que debían considerar los docentes para usar cada modelo y su relación con la experiencia de aprendizaje; también, las percepciones del profesorado descifradas en los comentarios más relevantes frente al modelo y la posible contribución a su práctica, como producto de la aplicación de una entrevista estructurada a los participantes, centradas en dos: ventajas y desventajas del uso en las prácticas de Enseñanza, así como los obstáculos para implementar estos modelos en la institución. 
Tabla 6 - Prototipos implementados por modelos didáctico con mediación TIC y evaluación de percepción

\begin{tabular}{|c|c|c|c|c|}
\hline \multirow{4}{*}{$\begin{array}{l}\text { Prototipos } \\
\text { implementados } \\
\text { por modelo } \\
\text { de mediación } \\
\text { didáctico }\end{array}$} & \multirow{2}{*}{\multicolumn{2}{|c|}{$\begin{array}{c}\text { Cuestionario } \\
\text { Evaluar el aporte del } \\
\text { prototipo en la gestión } \\
\text { educativa/académica }\end{array}$}} & \multirow{2}{*}{\multicolumn{2}{|c|}{$\begin{array}{l}\text { Entrevista } \\
\begin{array}{c}\text { Reconocer los resultados y pertinencia para la } \\
\text { implementación en los contextos }\end{array}\end{array}$}} \\
\hline & & & & \\
\hline & \multirow{2}{*}{$\begin{array}{l}\text { \# de } \\
\text { prototipo } \\
\text { por } \\
\text { modelo }\end{array}$} & \multirow{2}{*}{ 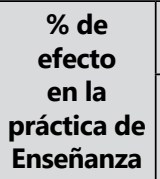 } & \multicolumn{2}{|c|}{$\begin{array}{c}\text { Percepción en los procesos de Enseñanza e } \\
\text { implementación en la institución }\end{array}$} \\
\hline & & & Ventajas / Desventajas & $\begin{array}{c}\text { Obstáculos de } \\
\text { implementación } \\
\text { institucional }\end{array}$ \\
\hline $\begin{array}{l}\text { Modelo de } \\
\text { integración } \\
\text { didáctico con } \\
\text { TIC centrado en } \\
\text { las técnicas de } \\
\text { Enseñanza }\end{array}$ & 4 & $70 \% / 100 \%$ & $\begin{array}{c}\text { Reconocieron las } \\
\text { bondades que brinda } \\
\text { este modelo a partir } \\
\text { de la autogestión del } \\
\text { aprendizaje, producto de } \\
\text { la simulación centradas } \\
\text { en experimentar } \\
\text { situaciones reales } \\
\text { (Second Life, realidad } \\
\text { aumentada o video } \\
\text { juegos) }\end{array}$ & $\begin{array}{l}\text { En su gran mayoría, } \\
\text { requieren conectividad, } \\
\text { así como el uso de } \\
\text { un dispositivo para } \\
\text { cada participante, } \\
\text { aspecto con el que no } \\
\text { se cuenta en todas las } \\
\text { instituciones }\end{array}$ \\
\hline $\begin{array}{l}\text { Modelo de } \\
\text { integración } \\
\text { didáctico con } \\
\text { TIC a partir de } \\
\text { los ambientes } \\
\text { de aprendizaje }\end{array}$ & 9 & $90 \% / 100 \%$ & $\begin{array}{c}\text { Sus estructuras a } \\
\text { partir de secuencias o } \\
\text { esquemas organizativos } \\
\text { por cursos, contenidos } \\
\text { (módulos o temas), } \\
\text { periodos temporales } \\
\text { (semanas o unidades) } \\
\text { para abordar } \\
\text { problemáticas } \\
\text { socioculturales reales } \\
\text { que aporta a la } \\
\text { formación de un perfil } \\
\text { de ciudadano crítico, } \\
\text { reflexivo y participativo }\end{array}$ & $\begin{array}{c}\text { Plantearon que } \\
\text { desconocían la } \\
\text { intención de algunas } \\
\text { metodologías } \\
\text { propuestas en los } \\
\text { AVA, así como la } \\
\text { secuencia didáctica } \\
\text { que debería contener } \\
\text { cada una de las } \\
\text { mismas. Plantean que } \\
\text { crear estas secuencias } \\
\text { requiere bastante } \\
\text { tiempo, conocimientos } \\
\text { didácticos más } \\
\text { profundos y formación } \\
\text { en competencias } \\
\text { digitales }\end{array}$ \\
\hline $\begin{array}{l}\text { Modelo de } \\
\text { integración } \\
\text { con TIC } \\
\text { centrado en los } \\
\text { componentes } \\
\text { cognitivos } \\
\text { necesarios para } \\
\text { la Enseñanza- } \\
\text { aprendizaje }\end{array}$ & 2 & $50 \% / 100 \%$ & $\begin{array}{c}\text { Mejora en la inclusión } \\
\text { a partir del uso de } \\
\text { propuestas que } \\
\text { permite el desarrollo } \\
\text { de habilidades, } \\
\text { competencias y hábitos } \\
\text { que le permitan resolver } \\
\text { problemas desde saberes } \\
\text { específicos flexibles } \\
\text { según la población a } \\
\text { quien va dirigido }\end{array}$ & $\begin{array}{c}\text { Falta formación } \\
\text { didáctica en temas } \\
\text { como la inclusión } \\
\text { educativa para } \\
\text { la población con } \\
\text { necesidades educativas } \\
\text { especiales }\end{array}$ \\
\hline
\end{tabular}

Fuente: Elaboración propia (2020) 
Estos resultados evidencian que un 70\% de los profesores (63) del 100\% participante (90) reconocieron las bondades que brinda el modelo de integración didáctico con TIC, centrado en las técnicas de Enseñanza y la autogestión del aprendizaje. No obstante, consideran que uno de los obstáculos a vencer es lograr que las instituciones gestionen la adquisición de dispositivos y conectividad para una implementación efectiva de lo propuesto; es decir, es un problema de gestión administrativa que tiene un impacto sobre las decisiones didácticas que asume el profesorado.

Sobre el modelo de integración didáctico con TIC centrado en los ambientes de aprendizaje, un $90 \%$ de los profesores (81) del 100\% participante reconocieron el valor del uso didáctico de estos AA, como escenarios de aprendizaje que permite aprender en contexto y usar el currículo y sus contenidos para resolver problemas reales. En la entrevista, plantearon como obstáculo la falta de formación didáctica en metodologías, experiencias y competencias digitales, lo que genera una dificultad en su implementación; rescatan valor de esta competencia en las prácticas de Enseñanza.

Finalmente, frente al modelo de integración con TIC centrado en los componentes cognitivos necesarios para la Enseñanza-aprendizaje, un 50\% de los profesores (45) reconocieron el valor que tiene este tipo de modelos en el desarrollo de habilidades, competencias y hábitos para resolver problemas desde saberes específicos y algorítmicos del área. Como obstáculo se encontró un desconocimiento frente a temas como la Educación inclusiva y estrategias didácticas con o sin TIC para su implementación. Los resultados finales de estas investigaciones permitieron generar las siguientes conclusiones:

- El control de variables demostró que la gestión de proyectos de innovación educativa para la implementación de modelos de integración didácticos mediados por TIC favorece parcialmente la cualificación de las prácticas de Enseñanza. Esto obedece a los obstáculos institucionales declarados por el profesorado en las entrevistas; también, a variables extrañas como la baja cantidad de dispositivos con las que cuenta la institución y las dificultades en la conectividad.

- Otro de los elementos que afectaron el control de variables y que fortaleció las variables extrañas, se focalizó en la débil formación del profesorado en conocimientos didácticos para identificar criterios de uso de cada modelo y competencias digitales para implementar prácticas de Enseñanza con TIC. 
- Los resultados de investigación permitieron corroborar la hipótesis alternativa (Ha), que declara que la gestión de proyectos de innovación educativa para la implementación de modelos de integración didácticos mediados por TIC favorece parcialmente la cualificación de las prácticas de Enseñanza. Superar las variables extrañas descritas y ajenas al profesorado implica una gestión institucional donde se evalúe el impacto de esta propuesta en el marco de investigación de corte longitudinal, desde una ventana de observación de tiempo que oscile entre 3 a 5 años y que permita monitorear indicadores de seguimiento. Sin duda, es una de las prospectivas que dan paso a nuevas investigaciones sobre el tema.

\subsection{Discusión: ¿cómo gestionar el proyecto educativo?}

Posterior a estos resultados, queda la pregunta por ¿cuáles son las fases que permite que este tipo de proyectos experimente una gestión que los lleve a arraigarse en los contextos educativos? En este sentido, la "Guía para el seguimiento y la evaluación de proyectos" (FEDERACIÓN INTERNACIONAL DE SOCIEDADES DE LA CRUZ ROJA Y DE LA MEDIA LUNA ROJA, 2011) recoge los principales cuestionamientos que conviene plantear para recoger las apreciaciones que deben proyectarse sobre la comunidad: efecto, eficacia y eficacia del uso de las TIC para la Enseñanza. Al respecto, el interés debe centrarse en monitorear los cambios logrados con la implementación de este tipo de propuestas (Figura 3).

Figura 3 - Guía para el seguimiento y la evaluación de proyectos educativos

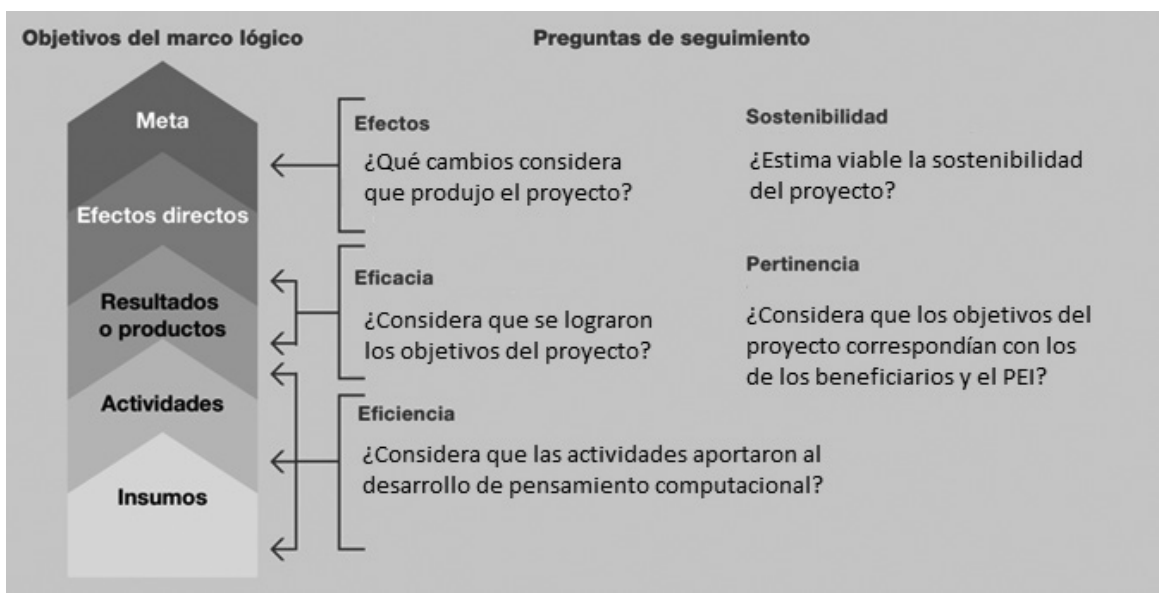

Fuente: IFRC (2011, p. 14) 
Respecto a la eficiencia en la implementación de modelos didácticos con mediación TIC, se puede decir que sus aportes se evidenciaron en la creación y testeo de diversos prototipos y diversos ambientes de aprendizaje (JIMÉNEZ-BECERRA; SALAMANCA ESPINOSA; LÓPEZ LÓPEZ, 2018), así como en el uso de los recursos (JIMÉNEZ-BECERRA, 2020B) que demostraron el efecto del valor de la planeación y análisis de las decisiones didácticas que debe tomar el profesorado en aspectos como la ruta como los resultados de aprendizaje esperados y el valor de las TIC en ellas. A partir de lo anterior, se evaluó la eficacia de los modelos propuestos al evaluar los tipos de intervenciones didácticas generadas en cada modelo, destacando la importancia de caracterizar -para la Enseñanza- las habilidades o capacidades propias del pensamiento social (PAGÉS, 1994) así como los criterios de selección para su efecto en la Enseñanza. También se identificó cómo el uso de los modelos con mediación TIC fomenta, en los estudiantes, la curiosidad, capacidad para considerar y buscar soluciones alternativas y originales a los problemas, que deben conocer las razones para estudiar determinadas temáticas y bajo determinada manera, dentro de situaciones concretas.

Esto permite concluir que, aunque en el diagnóstico fueron identificadas en el profesorado débiles competencias respecto al reconocimiento y manejo de las TIC, su interés para cualificarse a partir del uso de modelos didácticos utilizando este andamiaje y los criterios de uso y efecto para optimizar los procesos de aprendizaje aportaron en el desarrollo y fortalecimiento de competencias didácticas y tecnológicas.

Estas conclusiones emergen de la aplicación de instrumentos de recolección de datos mediante cuestionarios, grupos focales y entrevistas (JIMÉNEZ-BECERRA, 2020b), que permitió conocer la evaluación (REMOLINA-CAVIEDES, 2016), los alcances del proyecto y la percepción de su implementación por parte de los actores participantes de la comunidad educativa, permitiendo medir los indicadores de eficiencia, eficacia y efecto del proyecto, así como componentes para afirmar si se logra una verdadera innovación educativa a partir de la postura generada sobre este tema en esta investigación: mejorar para transformar... transformar para innovar, indicadores que monitoreen la pertinencia y la sostenibilidad a esa verdadera mejora.

Finalmente, es clave recordar que dentro de esta innovación, la inmersión de las TIC (JIMÉNEZ-BECERRA, 2018), responsable del origen de dichos nichos de aprendizaje a los que ahora se enfrenta la escuela, supone investigar, crear e implementar nuevos modelos innovación didáctica. Dichos modelos son definidos 
por Salinas Ibajnez (2004) como "una forma creativa de selección, organización y utilización de los recursos humanos y materiales, nueva y propia, que dé como resultado el logro de objetivos previamente marcados" (p. 10), en la que es indispensable articular las TIC y los saberes didácticos ¿estamos preparados, como profesores, para afrontar tal desafío? 


\title{
Evaluation of the educational management process for the integration of didactic models mediated by ICT: a multiple case study
}

\begin{abstract}
The article presents a study aimed at evaluating the effect of the implementation of ICTmediated teaching models on teaching practices in some Colombian educational contexts, from an investigation of mixed nature framed in the designs of Guskey to evaluate the effect and the single multiple case study to analyze the report of these models in learning. We worked with 35 Colombian teachers from schools and universities located in rural and urban geographical areas that belong to settings with economic limitations and social realities marked by the conflict. In the conclusions, it was possible to corroborate the alternative hypothesis (Ha): the management of educational innovation projects partially favors the qualification of Teaching practices. This, as a product of strange variables related to the low number of devices that the institution has and the weak didactic knowledge that teachers have for the use of technologies in the classroom.
\end{abstract}

Keywords: Didactic Models. Educational Management. Teaching Practices. Evaluation.

\section{Avaliação do processo educacional de gestão para a integração de modelos didáticos mediados pela TIC: um estudo de múltiplos casos}

\section{Resumo}

O artigo apresenta um estudo que objetiva avaliar o efeito da implementação de modelos didáticos mediados pelas TIC nas práticas de ensino em alguns contextos educacionais colombianos. A partir de uma investigação de natureza mista enquadrada nos desenhos de Guskey para avaliar o efeito e o estudo de múltiplos casos para analisar a contribuição desses modelos no aprendizado. Trabalhamos com 35 professores colombianos de escolas e universidades localizadas em áreas geográficas rurais e urbanas, caracterizadas por pertencer a cenários com limitações econômicas e com realidades sociais marcadas pelo conflito. Nas conclusões, foi possivel corroborar a hipótese alternativa (Ha), que afirma que o gerenciamento de projetos de inovação educacional favorece parcialmente a qualificação das práticas de ensino; isso como produto de variáveis estranhas no baixo número de dispositivos que a instituição possui e no fraco conhecimento didático que os professores possuem para o uso de tecnologias.

Palavras-chave: Modelos Didáticos. Gestão Educacional. Práticas de Ensino. Avaliação. 


\section{Referencias}

ALDANA, M.; BUITRAGO, J. Estrategias didácticas mediadas por lego ${ }^{\circledR}$ mindstorms ${ }^{\circledR}$ para desarrollar el pensamiento lógico para la programación en los estudiantes de la facultad de ingeniería de la Universidad del Quindío. Tesis (Maestría en Informática Eduacativa) Universidad de La Sabana, Chía, Colombia, 2019.

BRASLAVSKY, C. Bases, orientaciones y criterios para el diseño de programas de formación de profesores. Revista Iberoamericana de Educación, Madrid, v. 19, p. 13-50, ene-abr. 1999.

CARDELLI, J. J.; DUHALDE, M. A. Formación docente en América Latina: una perspectiva político-pedagógica. Cuadernos de Pedagogía, Barcelona, v. 308, p. 38-45, dic. 2001.

CASALLAS, A. Influencia de un entorno personal de aprendizaje en procesos de búsqueda, selección y evaluación de información para los proyectos de investigación de los estudiantes del curso 1002 del Colegio Marco Antonio Carreño Silva IED. Tesis (Maestría en Informática Eduacativa) - Universidad de La Sabana, Chía, Colombia, 2016.

CHITIVA, J.; TORRES, H. Implementación de un programa de aprovechamiento positivo del tiempo libre mediado por TIC en los estudiantes de la básica secundaria y media de la Institución Educativa Fonquetá del municipio de Chía, Cundinamarca. Tesis (Maestría en Proyectos Educativos mediados por TIC) - Universidad de la Sabana Chía, Colombia, 2018.

COLL SALVADOR, C. El currículo escolar en el marco de la nueva ecología del aprendizaje. Aula de Innovación Educativa, [s. 1.], n. 219, p. 31-36, 2013.

DONG, C.; NEWMAN, L. Ready, steady ... pause: integrating ICT into Shanghai preschools. International Journal of Early Years Education, London, v. 24, n. 2, p. 224-237, 2016. https://doi.org/10.1080/09669760.2016.1144048

ERICSON, B. Creación de Materiales para Recursos Educativos Digitales Abiertos (REDA): una estrategia de aprendizaje por proyectos que aporta al desarrollo de pensamiento computacional en el ciclo de educación media en la escuela normal superior de Leticia. Tesis (Maestría en Proyectos Educativos mediados por TIC) - Universidad de La Sabana, Chía, Colombia, 2018. 
FEDERACIÓN INTERNACIONAL DE SOCIEDADES DE LA CRUZ ROJA Y DE LA MEDIA LUNA ROJA. Guía para el seguimiento y la evaluación de proyectos y programas. Ginebra: Federación Internacional de Sociedades de la Cruz Roja y de la Media Luna Roja, 2011. Era IFRC

GÓMEZ, M. Propuesta pedagógica mediada por TIC para fortalecer el proceso de lectura y escritura inicial en estudiantes con diagnóstico con necesidades educativas especiales. Tesis (Maestría en Inforrmática Educativa) - Universidad de La Sabana, Chía, Colombia, 2018.

GUSKEY, T. Staff development and the process of teacher change. Educational Researcher, [s. 1.], v. 15, n. 5, p. 5-12, 1986. https://doi.org/10.3102/0013189X015005005

HERRERA, M.; FERNÁNDEZ, C.; SEGUEL, R. Percepción de los profesores sobre integración de TIC en las prácticas de enseñanza en relación con los marcos normativos para la profesión docente en Chile. Ensaio: Avaliação e Políticas Públicas em Educação, Rio de Janeiro, v. 26, n. 98, p. 163-184, jan./mar. 2018. https://doi.org/10.1590/s0104-40362017002501119

JACOBY, K.; MORA, D. Prácticas y ejercicios: la teoría del proyecto (marco lógico, cadenas de impacto). In: PROGRAMA DE CAPACITACIÓN EN EVALUACIÓN DE IMPACTOS. SEMINARIO DE POSTGRADO IMPARTIDO EN LA ESCUELA DE ADMINISTRACIÓN PÚBLICA DE LA UNIVERSIDAD DE COSTA RICA. Anais[...] San José, Costa Rica: 2008

JIMÉNEZ -BECERRA, I. Rasgos y tendencias de la Didáctica con TIC: retos a partir de la nueva ecología del aprendizaje. Estudios Pedagógicos, Valdivia, v. 46, n. 2, p. 215-229, 2020b. https://doi.org/10.4067/S0718-07052020000200215

JIMÉNZ-BECERRA, I. Triángulo lógico: una ecuación didáctica emergente para aprender metodología de la investigación. Chía: Universidad de La Sabana, 2020a.

JIMÉNEZ-BECERRA, I.; ESCOBAR, C. Uso didáctico del videojuego educativo para la enseñanza de las ciencias sociales: un estado del arte. Paideia, Concepción, n. 58, p. 53-70, 2016. 
JIMÉNEZ-BECERRA, I.; FERNÁNDEZ PALMA, O. E.; ALMENÁREZ MORENO, F. T. Diseño pedagógico adaptativo para el desarrollo de MOOC: una estrategia para el desarrollo de competencias en contextos corporativos. Revista Electrónica de Investigación Educativa, Ensenada, v. 22, e16, 2020. https://doi.org/10.24320/redie.2020.22.e16.2192

\section{JIMÉNEZ-BECERRA, I.; SALAMANCA ESPINOSA, L.; LÓPEZ LÓPEZ,}

L. G. Implementación de entornos personales de aprendizaje para fortalecer las habilidades comunicativas. Revista de la Universidad Pedagógica Nacional. Facultad de Humanidades, Bogotá, v. 47, n. 11, p. 119-132, ene./jun; 2018. https://doi.org/10.17227/folios.47-7401

JIMÉNEZ-BECERRA, I.; SEGOVIA-CIFUENTES, Y.-M. Modelos de integración didáctica con mediación TIC: algunos retos de innovación en las prácticas de enseñanza. Revista: Culture and Education. [s. 1.], v. 32, n. 3, p. 399-440, sep. 2020. https://doi.org/10.1080/11356405.2020.1785140

KAPLAN, A.; HAENLEIN, M. Higher education and the digital revolution: about MOOCs, SPOCs, social media, and the Cookie Monster. Business Horizons, v. 59, n. 4, p. 441-450, jul./ago. 2016. https://doi.org/10.1016/j.bushor.2016.03.008

LONDOÑO, C.; RUBIANO, L.; TRIANA, C. Integración curricular de las TIC a través del componente "estrategia pedagógica del modelo mític@" mediante buenas prácticas pedagógicas de la Institución Educativa Diosa Chía. Tesis (Maestría en Proyectos Educativos mediados por TIC) Universidad de La Sabana, Chía, Colombia, 2018.

LUGO, M.; KELLY, V. La Matriz de las TIC: una herramienta para planificar las tecnologías de la información y la comunicación en las instituciones educativas. Buenos Aires: Unesco, 2011.

MARTÍNEZ, R.; FERNÁNDEZ, A. Medotologías e instrumentos para la formulación, evaluación y monitoreo de programas sociales. Ciudad de México: Cepal, 2008.

MILLER, R.; SHAPIRO, H.; HILDING-HAMANN, K. School's over: learning spaces in Europe in 2020: an imagining exercise on the future of learning. Sevilla: European Commission, 2008.

PAGÈS, J. La didáctica de las Ciencias Sociales, el currículum y la formación del profesorado. Signos Teoría y Práctica de la Educación, [s. 1.], v. 5, n. 13, p. 38-51, 1994. 
REMOLINA-CAVIEDES, J.-F. Evaluación educativa y codeterminaciones de la producción escolar. Ensaio: Avaliação e Políticas Públicas em Educação, Rio de Janeiro, v. 28, p. 135-155, jan./mar. 2016. https://doi.org/10.1590/s0104-40362019002701629

SALINAS IBA]NEZ, J. M. Cambios metodológicos con las TIC: estrategias didácticas y entornos virtuales de enseñanza-aprendizaje. Bordón, Madrid, v. 56, n. 3, p. 469-481, 2004.

SÁNCHEZ, V.; GALVIS, C. Aprendizaje para la comprensión: estrategias didácticas para fomentar el pensamiento matemático a partir de un ambiente de aprendizaje colaborativo mediado por TIC en los IED San Francisco y Manuelita Sáenz. Tesis (Maestría en Proyectos Educativos mediados por TIC) - Universidad de La Sabana, Chía, Colombia, 2017.

STAKE, R. Investigación con estudio de casos. Madrid: Ediciones Morata, 1999.

SUMMO, V.; VOISIN, S.; TÉLLEZ-MÉNDEZ, B.-A. Creatividad: eje de la educación del siglo XXI. Revista Iberoamericana de Educación Superior, México, v. 7, n. 18, p. 83-98, ene. 2016.

TERRIBILI FILHO, A. A profissionalização do gerenciamento de projetos no Brasil: um estudo comparativo dos benchmarkings de 2004 e 2010. Revista Estratégica, São Paulo, v. 11, n. 1, p. 9-20, 2011.

VILLARREAL, D. Recurso educativo digital adaptativo "el rescate del reino": una estrategia para aprender permutaciones sin repetición. Tesis (Maestría en Proyectos Educativos mediados por TIC) - Universidad de La Sabana, Chía, Colombia, 2016.

VOGLER, J.; BLANCHARD, S.; FIJALKOW, J. La evaluación. Paris: Hachette, 1996.

WESTRICK, J.; MORRIS, G. Pedagogía de la formación del profesorado: interrumpiendo el aprendizaje de la observación. Enseñanza de la Educación, [s. 1.], v. 27, n. 2, p. 156-172, 2016. 


\section{Información de autores}

Isabel Jiménez Becerra: Doctora Cum Laude en Educación en la Línea Didáctica de la Historia, la Geografía y las Ciencias Sociales de la Universidad Autónoma de Barcelona. Profesora categoría Asistente, Investigadora perteneciente al Grupo de investigación Proventus, Tecnologías para La Academia y Grupo de Investigación ÍCONO de la Institución Universitaria Pascual Bravo. Contacto: isa.pedagoga2032@gmail.com

iD https://orcid.org/0000-0003-4263-1970

Kemel A. Ghotme: Profesor Asistente de la Universidad de La Sabana. Director del Grupo de investigación Translational Neuroscience Research Lab. Pediatric Neurosurgeon Department of Neurosurgery, Fundación Santa Fe de Bogotá. Contacto: kemel.ghotme@ unisabana.edu.co

iD https://orcid.org/0000-0001-6802-4373

Artieres Estevao Romeiro: Docente e investigador del Departamento de Ciencias de la Educación, Grupo de investigación Teoría Crítica y Educación, Universidad Técnica Particular de Loja. Contacto: aeromeiro@utpl.edu.ec

iD https://orcid.org/0000-0002-6219-2867

Lina Rosa Parra Bernal: Doctora en Educación de la Universidad de la Salle. Investigadora del Grupo de Investigación Educación y Formación de la Universidad Católica de Manizales. Contacto: lrparra@ucm.edu.co

iD https://orcid.org/0000-0001-9838-4783 